Journal of Engineering and Applied Sciences 14 (Special Issue 2): 5429-5436, 2019

ISSN: 1816-949X

(C) Medwell Journals, 2019

\title{
Effect of Ferroelectric Nanoparticles on Electro Optic Properties of Polysiloxane Liquid Crystal
}

\author{
${ }^{1}$ Khalid Al. Ammar and ${ }^{2}$ Sura Al. Asadi \\ ${ }^{1}$ Department of Physics, College of Education for Pure Sciences, University of Babylon, Hillah, Iraq \\ ${ }^{2}$ General Directorate of Education in Babylon, Ministry of Education, Baghdad, Iraq
}

\begin{abstract}
In the present research influence found that adding Sn2P2S6 (SPS) nanoparticles on the electro optic properties of poly-siloxanes side chain liquid crystal polymers have been studied. The five samples of different spacer length have been used. It is found that switching voltage increases with spacer length increasing. The switching times (on) and (off) have been measured through the period between planer and homotropic alignment which happens under the electrical field effect. The variation of the threshold voltage is measured at constant steps below nematic isotropic transition Temperature $\left(\mathrm{T}_{\mathrm{NI}}\right)$ for series of polymers. It is found that the high spacer length of polymers have great threshold voltage of the electro-optic response and this is related to the intrinsic elastic constant of the liquid crystal polymers. Infrared dichroism measurements have been used to determine the orientational order parameters of the liquid crystalline side chain polymers. By identifying a certain band (CN stretching) in the infrared absorption spectrum, it is found that the orientational order depends on the temperature with different spacer length. A simple model is used to indicates the origion of the effects of observed which appear to arises from the constraint offered by the coupling of the mesogenic units to the polymer backbone.
\end{abstract}

Key words: The spacer length polysiloxane, liquid crystal polymer, electro optic properties of matter, SPS, CN stretching, temperature

\section{INTRODUCTION}

Liquid crystal property is the description of the intermediate situation that is confined configured in the range between solid-state where the movement of molecules bound with high system (orientation) and between states isotropic liquid in which the molecules owns freedom of movement (no orientation) and it is found between them by thermal or cold action or by the influence of asolvent. They are viscous, jelly-like materials that resemble liquids in certain aspects. Liquid crystals must be geometrically highly anisotropic (having different optical properties indifferent directions). Side chain liquid crystal polymers are acomposite from of macromolecule built up from three basic types of molecular unit. These are the polymer backbone, the mesogenic units and the coupling chains which link the mesogenic unit to the polymer chain. A large number of side chain polymers in which the nature of these three components and their interconnection are varied have been synthesised and studied (McArdle, 1989; Plate and Shibave, 1987). They are classified as main-chain LCP where mesogenic groups are part of the polymer backbone. Another approach was to link the rigid group to apolymer backbone as side chains (side-chain LCP) (Zentel, 1998). No rigid segments are present in the polymer main chain usually flexible, so, backbone of the SCLCP's contains on tilt suitable mesogenic groups with main chain for formation statistical random-coil. mesogenic groups, these groups restrict the conformational freedom of the main chain. The most crucial aspect is the union between the polymer main chain and the mesogenic side units when mesogenic units are directly attached to the polymer main chain, the tendency towards astatistically distributed chain conformation hinders any anisotropic orientation of the side unit. The importance of this effect is increased by the steric hindrance of the relatively voluminous structure that further decreases any orientational order. The influence can be decreased by the use of flexible spacers in between the polymer main chain and the mesogenic side groups. Due to the introduction of flexible spacers the motion of polymer main chain are decoupled to certain extent from the possible orientational order of the side groups in which the sign of the coupling may favour a positive or parallel arrangement simply because of the nematic field or the bias may be to a perpendicular or

Corresponding Author: Khalid Al. Ammar, Department of Physics, College of Education for Pure Sciences, University of Babylon, Hillah, Iraq 
negative arrangement of the side group and the polymer chain due to the particular geometry of the coupling chain. Small angle neutron scattering experiments have shown that for polyacrylate and polysiloxane based side chain liquid crystal polymers both positive (McArdle et al., 1990) and negative coupling (Noirez et al., 1988) are possible, although, the level of coupling in either case is relatively small. Studied involving cross-linked systems and fibres have shown that there is a regular alternation in the sign of the coupling for a porticular polyacrylate and polysiloxane based systems as the length of the coupling chain is increased. This contribution focuses on the influence of this coupling between the mesogenic side-group and the polymer backbone on the resultant electro-optic properties of such materials. In particular we centre our attention on the influence of the coupling chain upon the curvature elasticity which determines the ease with which the director pattern may be modified using external electric fields. A number of contributions concerned with these properties have appeared, for example, (Portugall et al., 1982; Ringsdorf and Zentel, 1982; Coles, 1988), although, none has considered the systematic variation of properties with changing coupling chain length for a series of polymers. We shall considered a series of polysiloxane based side-chain liquid crystal homopolymers in which the coupling chain length is systematically varied. Electro-optic measurements are used to evaluate the threshold voltages for this series of polymers. Although, the variation of threshold voltage with systematically changing coupling chain length correlates with spares variety of values for similar polysiloxane based polymers (Tal'Roze et al., 1983; Haase, 1989). Comparsion is made with existing models of curvature deformation in low molar mass liquid crystal systems. Where amore modern approach is the elastic continuum theory. The LC is considered as a continuum and molecular details are ignored (Leslie, 1992).

\section{MATERIALS AND METHODS}

Monomers of type Warner (1989), (where $\mathrm{n}=2$, 3,4 and 6) were prepared the methods Portugal et al. (1982). For the preparation of the monomer with $\mathrm{n}=5,5$-chloropentanol was prepared via reduction of the appropriate acid chloride with LiAIH4 (Vogel, 1978). The use of this route for the preparation of the monomer with $\mathrm{n} 4$ results in cyclisation of the 4-chiorobutanol and hence, gives poor yields for the first step. An alternative procedure to prepare the 4-(4-hydroxybutoxy) benzoic acid was used as detailed elsewhere (Whitcombe et al.,

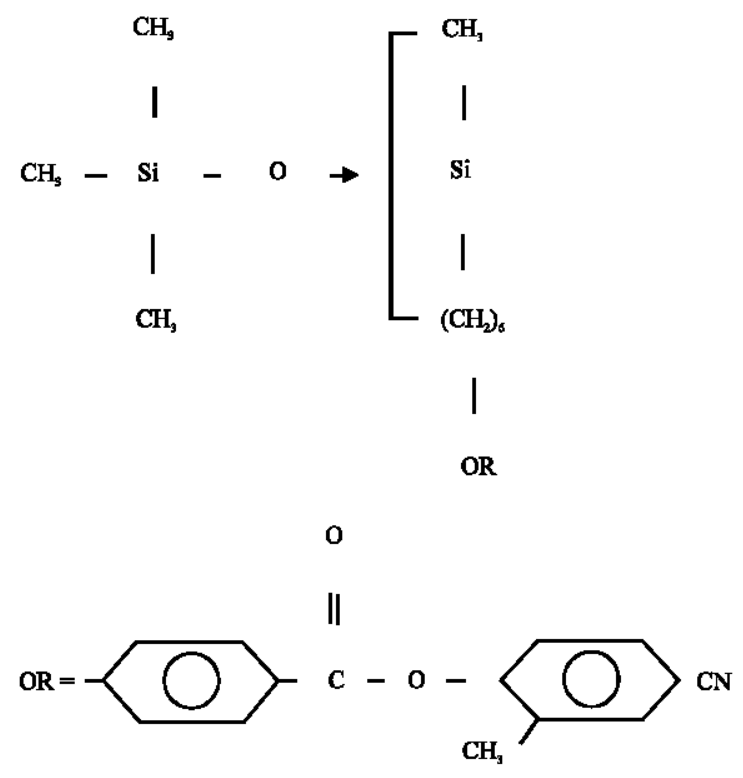

Fig. 1: Polymers show nematic phases

1991). Polymerization of these monomers was performed in deoxygenated chlorobenzene solutions under reduced pressure at $55^{\circ} \mathrm{C}$ with $1 \mathrm{~mol} \% \mathrm{AIBN}$ as the initiator. After $24 \mathrm{~h}$, the resulting polymers were collected by precipitation into methanol and purified by repeated precipitation into diethyl ether, followed by drying under reduced pressure for $48 \mathrm{~h}$ at $100^{\circ} \mathrm{C}$. The molecular weight data were obtained by GPC (RAPRA Ltd) at room temperature with tetrahydrofuran as the eluent. All of these polymers show nematic phases with well defined nematic-isotropic transition temperatures and $\mathrm{Sn}_{2} \mathrm{P}_{2} \mathrm{~S}_{6}$ (SPS) nanoparticles as shown in Fig. 1.

\section{Experimental work}

Cell preparation: Good alignment is necessary in polymer layers before electro optic properties can be measured. If this is not achieved, then the freedericksz transition is observed over arrange of applied field and meaningless data results. Therefore, efforts have been spent to achieve high quality fabricated electro-optic cells (Wangsoub and Mitchell, 2009). The typical construction of an electro optic cell used experimentally in these investigations. Electro-optic cells with predefined director orientation were constructed from patterned tin oxide coated glass slides (Baltracon Z20). The cell electrodes, after cleaning in an ultrasonic bath and drying were coated with a thin layer of a polyimide precursor consisting of a $5 \%$ solution of Rodehftal 322 in dimethyl formamide using a spin coater. These coated slides were then subjected to thermal cycles and a mechanical treatment using a cloth. 
The selected polymer sample was carefully applied onto one of the treated glass electrodes and the complete assembly was heated above the clearing point of the polymer in order to allow the trapped air to escape. The second glass electrode was then mounted above the first electrode using $0.025 \mathrm{~mm}$ thickness Kapton sheet as spacers. The quality of the prealignment treatment was confirmed through examination of each cell with a polarizing microscope. Complete and uniform director alignment was obtained by holding the completed electro-optic cell at a temperature $1^{\circ} \mathrm{C}$ measured clearing point for 10-24 h. This technique was successful in inducting a uniform director alignment within the

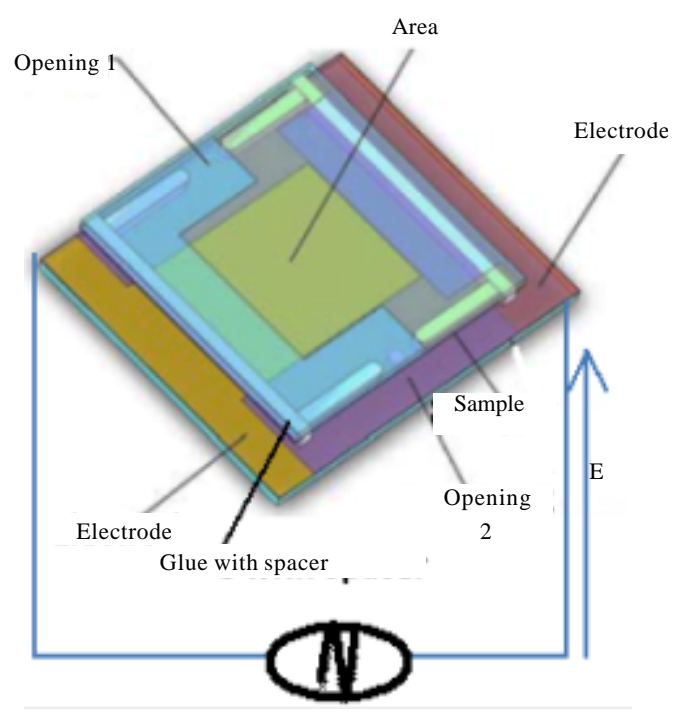

Fig. 2: The electro-optic cells prepared for all the materials electro-optic cells prepared for all the materials used in this research is shown in Fig. 2. In all cases the predefined director alignment was parallel to the electrode surface and to the direction of rubbing. The orientational order parameter $\mathrm{S}$ for the polymers in the series with $\mathrm{n}=4,5,6$ was measured as a function of temperature.

Electro-optic systems: The most important three devices were used in this research for electro optic measurement are HCS 402 (ALCT) and MK 1000 shown in Fig. 3. The Automatic Liquid Crystal measurement systems (ALCT) are USB based instruments which can be connected conveniently to both desktop and laptop computers. Depending on options, the (ALCT's) easy-to-use software allows for the measurement of material parameters for both positive and negative dielectric liquid crystals and material parameters of liquid crystal such as dielectric constants $\varepsilon \perp$ and $\varepsilon \|$, elastic constants splay and bend ( $\mathrm{K} 11$ and $\mathrm{K} 33$ ), voltage holding ratio measurement system, optical switching time, optical transmittance vs. voltage threshold voltage $\mathrm{Vc}$, polarization current Ip and viscosity, the (ALCT) can also be integrated with instec's microscope hot and cold stages and temperature controllers, enabling the user to analyze the temperature dependence of the above parameters. (HCS302) has the following features: dualheater microscope hot and cold stage for increased temperature gradient uniformity, programmable precision temperature control from $200-600^{\circ} \mathrm{C}$. Controlled fast heating and cooling rate. Large viewing aperture. Variable sample chamber height. Easy side sample loading with

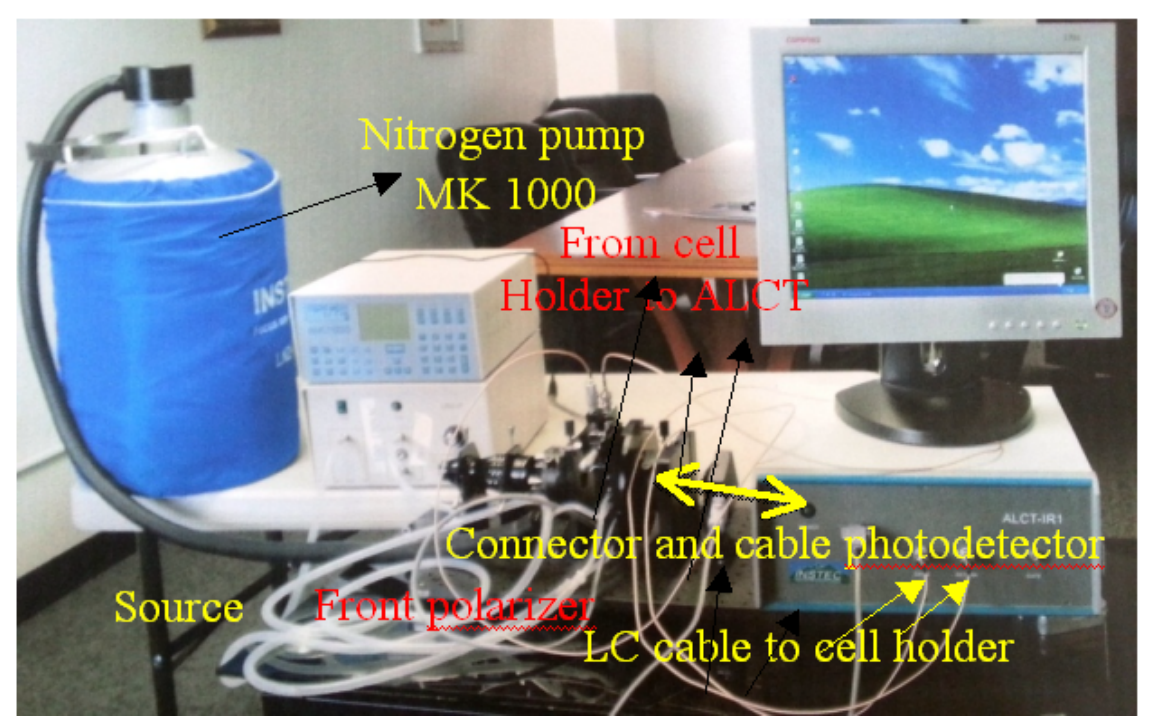

Fig. 3: Electro optic measurement are HCS 402 (ALCT) and MK 1000 


\section{J. Eng. Applied Sci., 14 (Special Issue 2): 5429-5436, 2019}

standard microscope slides. (The high precision temperature controller) (MK1000) represents a leap in performance and ease. It provides temperature control resolution and precision to $0.001^{\circ} \mathrm{C}$, accommodates both platinum( RTD) and individually calibrated thermistors as temperature sensors and can optionally control up to 4 instec hot and cold stages, plates or chucks. The front panel interface with $192 \times 128$ pixel graphic display allows easy stand alone programming and control while the standard (USB2.0) communication port and included software provides foor simple computer control. Optional (RS232) and (IEEE488) communication ports are also available. All connections to the (MK1000) use different types of ports eliminate the possibility of an incorrect connection a minimum configuration uses only the sensor/heater port for connecting the hot stage or hot chuck cable to and the line power input port for connection the power cable to additional connection for communications and cooling accessories are available. Depending do not connect power cable and turn on the (MK1000) until all other connections have been made. (MK1000) has the following features: accurate to $0.001^{\circ} \mathrm{C}$ temperature resolution and precision. Fully programmable without host computer. (USB2.0) communication port with win-temp software. Compatible with all instec hot and cold stages, chucks and plates. User adjustable ramp (rate of heating/cooling) to user set temperature point. Temperature control system includes (MK1000) controller, nitrogen container nitrogen pump (LN2-p) and hot-cooling stage. Temperature sensor (RTD) thermistor or thermocouple (for thermistor sensor, the heating limit is $250^{\circ} \mathrm{C}$ ) and operation environment: temperature $0 \sim 50^{\circ} \mathrm{C}$ humidity $<85 \% \mathrm{RH}$ with minimal electromagnetic interference. Dual pane window for better thermal isolation integrated aperture window defrost system inner lid for improved sample temperature uniformity, vertical and horizontal mounting, optional precision $\mathrm{X}-\mathrm{Y}$ micropositionar for sample positioning and application software win-temp allows remote control from host computer. Optical test bench subsystem. This includes light source, polarizer, rotatable hot-cooling stage holder analyzer and photo detector holder. This test bench allows user to: arrange polarizer and analyzer perpendicular and parallel to each other. Test cell in side of the hot-cooling stage can be rotated in full $360^{\circ}$ range. Light source, polarizer and analyzer are installed in sealed dark sections to prevent the contamination of optical components. Light sealable working chamber shields a way the room lighting.

\section{RESULTS AND DISCUSSION}

Static electro-optic properties: The dynamic electro optic properties are important for each member of the spacer length series of the side chain liquid crystal polymers. By using the experimental arrangement described in the previous study it was possible to determine the threshold electric field required to distort the surface prealigned director orientation. In order to achieve complete switching we typically applied (147-240) V (peak to peak) at a frequency of $500 \mathrm{~Hz}$. The variation of the transmitted light intensity as a function of the applied voltage and the required voltage for each material in this research for complete switching is shown Fig. 4a-e. For all polymers the dielectric anisotropy is positive. The electric field deforms an initial planar surface alignment and in this case of the geometry is dominated by the splay component of the curvature elasticity (K11) for small deformations. The threshold voltage $V$ th is related to the curvature elasticity. However, for a polymer system there are a number of particular problems involved in the evaluation of $\mathrm{Vth}$, each of which relates to the high viscosity of the polymer. The most important of which is ensuring that before starting any measurements the sample is in a complete equilibrium state. Although, there are some differences in the required voltage. Where Fig. 5a-c show switch on-time $\tau_{\text {on }}$ as a function of the time for which the field is switched off before the $\tau_{\text {off }}$ measurement. Each set of experiments was carried out at fixed temperature. All samples show similar curves, although, of course the time scales vary and these curves show several characteristic features. The purpose of the reducing the temperature below isotropic transition temperature is reached to saturation state. Implemented a cyclic experiment in which using the values of $\tau_{\text {on }}$ to determine when a steady state is reached. The method involves applying an electric field, measuring $\tau_{\text {on }}$, removing the field, waiting for a time $\tau_{\text {off }}$ and then repeating the cycle. When $\tau_{\text {off }}$ reaches and exceeds the true relaxation time for the polymer system, then $\tau_{\text {on }}$ will show reproducible values. The approach allows both $\tau_{\text {on }}$ and $\tau_{\text {off }}$ to be evaluated (Blinov, 1983). The reproducible switching effect were observed at temperatures close to the nematic- isotropic transition temperature $\mathrm{T}_{\mathrm{NI}}$ where the viscosity of the polymer is relatively low and measurement were made in the range of $\mathrm{T}_{\mathrm{NI}^{-}}-\mathrm{T}_{\mathrm{NI}}-3$ for polymers one and two and no response to the applied field had been observed for these materials in the smectic phase but measurements were made for the polymers 3-5 in the range of $\mathrm{T}_{\mathrm{IN}}-\mathrm{T}_{\mathrm{NI}}-5$ at the same time reproducible switching effects were observed in the smectic phase for polymer 3. Again the voltages for the complete orientation are applied to the cell and calculates the required time to the occurrence of complete orientation between high and minimum intensity at different temperatures. An alternative approach which is the more standardmethod reported in the research is to measure 

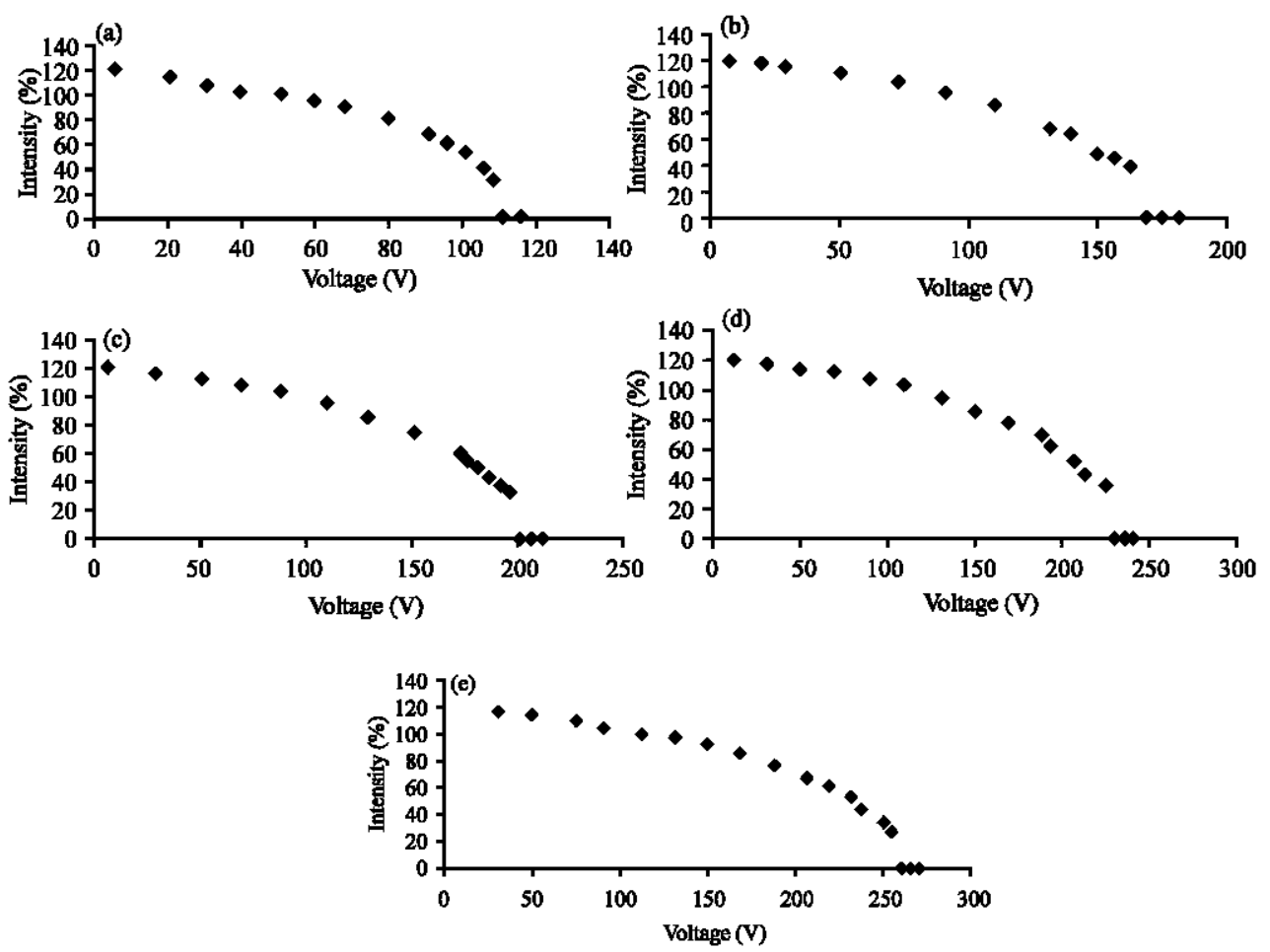

Fig. 4: a-e) The applied voltage and the required voltage for each material; Polymer 1-5

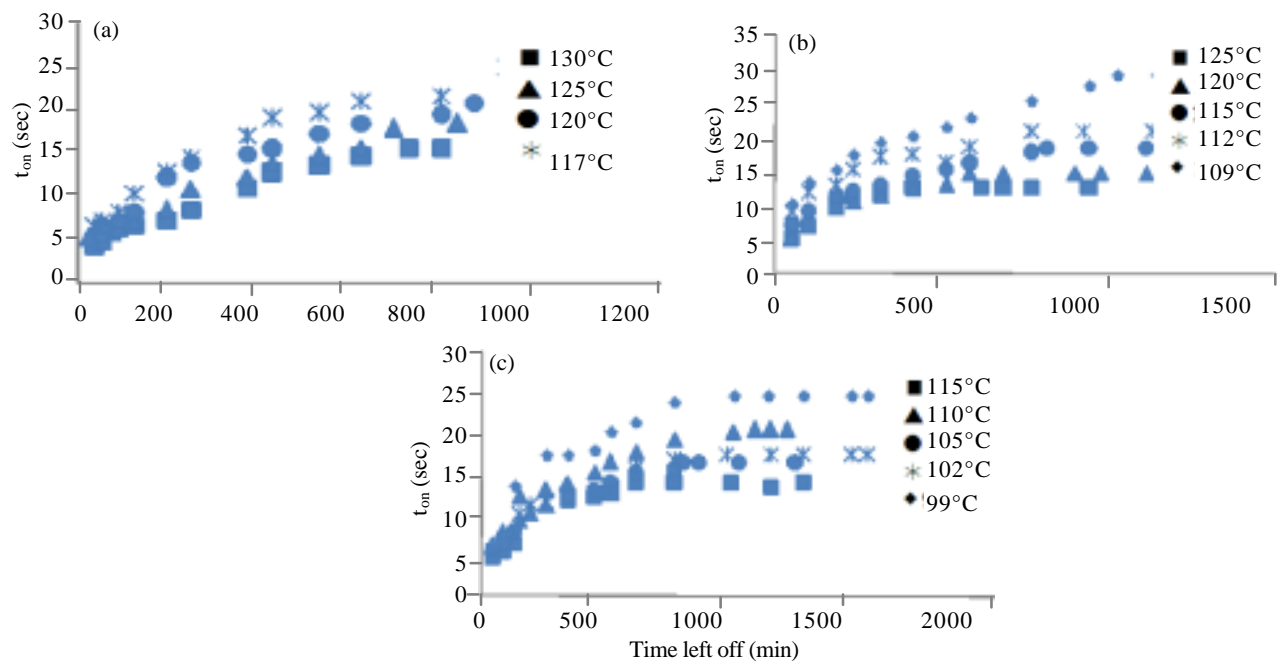

Fig. 5: Switch on-time $\tau_{\text {on }}$ as a function of the time for which the field is switched off before the $\tau_{\text {off }}$ measurement; a) P5; b) P4 and P3

the exponential decay of the transmitted light signal after the field is removed. As indicated above this is much more a measure of the state of alignment of the mesogenic units and may not indicate the state of the polymer chains. For each of the samples considered and for each temperature of measurement we have establish the real relaxation time of the polymer system through the use of a cyclic electro-optic procedure. In Fig. 6, it can be seen in general, the decreases spacer length leads to decreases of temperature $\mathrm{T}$ and increases of the relaxation time $\tau_{\text {off }}$ and it can be seen in single polymer increases of Temperature $\mathrm{T}$ leads to decreases of the relaxation time $\tau_{\text {off }}$ because low viscosity. This figure expresses the cyclic experiment and show temperature dependence of $\tau_{\text {off }}$ with $T$. The differences between methods arise because in the cyclic experiments 


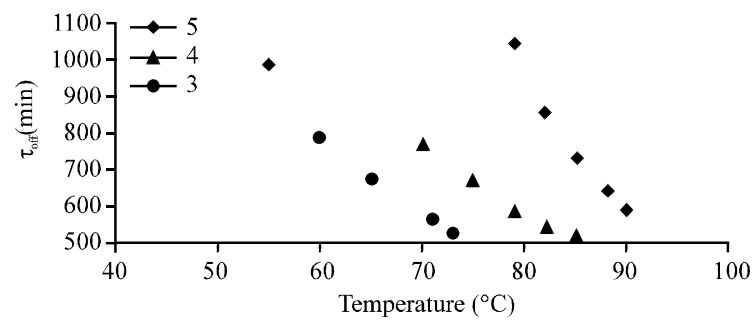

Fig. 6: $\mathrm{t}_{\mathrm{off}}$ variation as a function of $(\mathrm{T})$ at $\mathrm{tOn}$ and applied

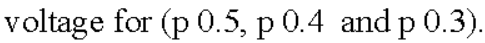

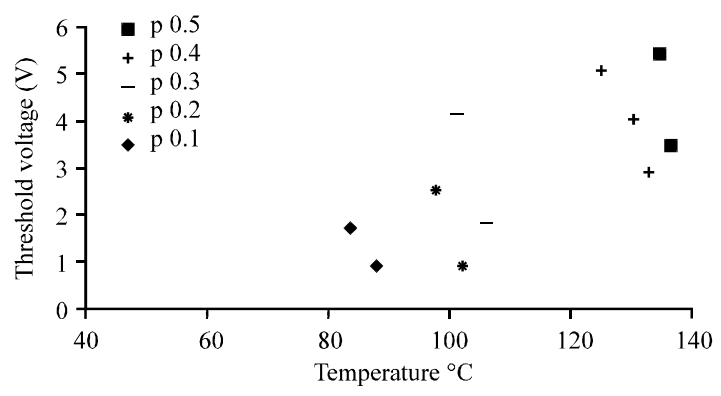

Fig. 7: Plots of the measured threshold voltages a function of temperature dichroism as a function of temperature

determining the equilibrium relaxation time by considering the switch-on time as a function of the allowed relaxation time, it reaches a plateau when both the polymer backbone and the mesogenic elements have relaxed and hence, the values of $\tau_{\text {off }}$ reported are those arising from the relaxation of a partially coupled system of the mesogenic and polymer backbone. Whereas in the direct measurement it is considering the relaxation to the initial light intensity level which does not necessarily indicate the equilibrium state of the whole system but merely that of the mesogenic units (AI-Ammar and Mitchell, 1992).

Relation ship between threshold voltage and the spacer length: In order to examine the role of the spacer length it is useful to eliminate the effects on the threshold voltage versus temperature curve of the differing transition temperatures. For this purpose the data shown in Fig. 7 may be mapped onto a universal function of the form: where $\alpha, \beta$ and $\mathrm{q}$ are constants. A fit is reasonable to take the dielectric anisotropy and as constant across the of polymers with differing coupling chain length. Of course there will be a slight increase (-15\% from n6-n2) due to the increasing number density of the mesogenic units and the fact that the remaining two components, namely the polymer backbone and the coupling chain probably make only an isotropic contribution. Such small approximation would allow us to make direct comparison between the different polymers in terms of the measured threshold voltages. If we make the correction for the change

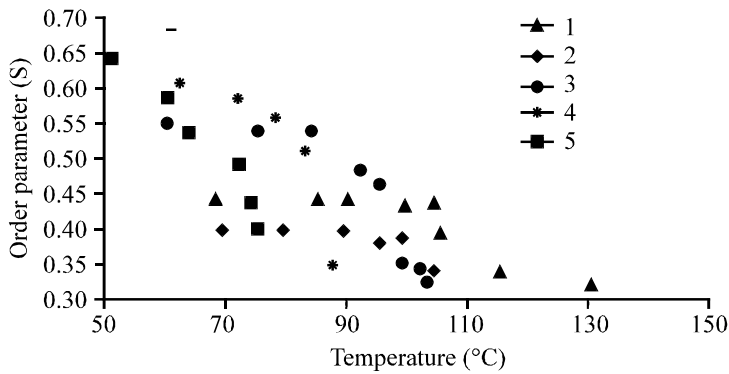

Fig. 8: The variation of the measured order parameter $\mathrm{S}$ obtained through measurement of the infra-red dichroism as a function of temperature.

in number density of the highly polarisable groups then this will enhance the trend of increasing threshold voltage with decreasing spacer length (Young, 1983).

Order parameter: In the present study, using infrared dichroism to analyze the orientational ordering of the liquid crystalline side-chain polymers on prealigned cells. Also, using the different molecular weights to obtain information about the orientational order of the head part by observing the bands corresponding to the vibrations of the relevant functional groups where the major vibration absorption band $\mathrm{C}=\mathrm{N}$ occur in the $2235 \mathrm{~cm}^{-1}$ region. Measurements were made for each sample over a range of temperatures to include both nematic phases, the resultant order parameter are displayed in Fig. 8. The variation of the polymer space length leads to distinctive trends in the order parameter versus temperature plots however, each individual curve has the same basic form (Young, 1983).

Curvature elasticity: Amicroscopic understanding of curvature elasticity remains a considerable challenge even in the area of low molar mass liquid crystalline materials. Existing approaches in the literature are very much first order relating either to the Maier-Saupe mean field theory (Vertogen and Jeu, 1988; Gennes, 1971). Or developed from the Landau-De Gennes expansion of free energy as a function of the order parameter (Vertogen and Jeu, 1988; Stode and Patron, 2007; Nehring et al., 2005). Saupe and Nebring use the Maier-Saupc approach to establish the relationship (Gennes, 1971; Mitchell et al., 1987):

$$
\mathrm{K}_{\mathrm{u}}=\frac{\mathrm{c}_{\mathrm{ii}} \mathrm{S}^{2}}{\mathrm{~V}_{\mathrm{m}}^{7 / 3}}
$$

Where:

$\mathrm{V}_{\mathrm{m}}=$ The molar volume

$\mathrm{c}_{\mathrm{ii}}=$ Temperature independent constant which is a function of the particular molecule or mesogenic unit 


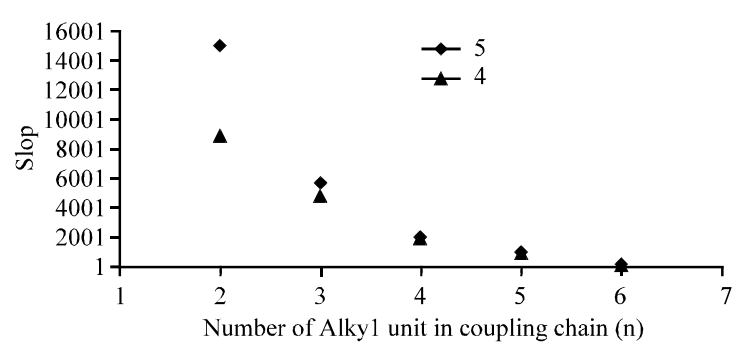

Fig. 8: We may relate the slope for each set of points to the intrinsic elastic constant cii shows we may relate the slope for each set of points to the intrinsic

In essence this approach groups all of tie temperature dependence into $\mathrm{S}_{2}$ and $\mathrm{V}_{\mathrm{m}}$, although, the latter term varies only slightly over the temperature range considered in this study. We can think of $c, 1$ as an intrinsic curvature elastic constant. If we take account of the dependence of b.c on S, we may combine Eq. 1 and 2 to give (Toyne, 1987):

$$
\mathrm{V}_{\mathrm{th}}^{2}=\frac{\mathrm{c}_{\mathrm{ii}} \mathrm{S}}{\mathrm{V}_{\mathrm{m}}^{7 / 3} \varepsilon \circ \varepsilon}
$$

where, $\Delta \varepsilon$ is an intrinsic dielectric anisotropy, i.e., that for a perfectly aligned system. If the variation in the threshold voltage with coupling chain length was due to differences in the level of orientational ordering. This is clearly not the case. We may relate the slope for each set of points to the intrinsic elastic constant $\mathrm{c}_{\mathrm{ii}}$ Fig. 9 shows a plot of these slopes against the coupling chain length $\mathrm{n}$ or more strictly the number of alkyl-groups in the spacer unit. The fact that there is a variation in the slope indicates that the coupling chain has a significant role in determining the curvature elastic constant. If we partition the slope into a component which is independent of $\mathrm{n}$ and a variable component, it is clear that the $\mathrm{n}$ independent term is small in comparison to the variable component. The strong variation in slope would appear to indicate that there is significant and changing coupling between the mesogenic side groups and the pollymer backbone (Saengsuman et al., 2007).

The chemical composition and spacer length of the most influential factors on the behaviour of the polymer and its switching times indicates that the material quickly changes. The variation of the coupling chain length in this series of siloxane based side chain liquid crystal polymers and $\mathrm{Sn}_{2} \mathrm{P}_{2} \mathrm{~S}_{6}$ (SPS) nanoparticles has impact upon both the phase behavior and upon the electro-optic properties. The increase in the nematic-isotropic transition temperature with increasing spacer length mirrors similar
Table 1: Molecular weight and phase transition data determined by using D.S.C** in Chemistry Department, University of Reading, U.K

\begin{tabular}{llllcc}
\hline Polymer & $\mathrm{n}$ & $\mathrm{Mn}$ & $\mathrm{Dp}$ & $\mathrm{Tg}^{0} \mathrm{c}$ & $\mathrm{Tni}^{0} \mathrm{c}$ \\
\hline 1 & 6 & $5.1 \times 105$ & 180 & 39 & 135 \\
2 & 5 & $2.0 \times 105$ & 100 & 40 & 130 \\
3 & 4 & $2 \times 105$ & 60 & 45 & 120 \\
4 & 3 & $3.2 \times 105$ & 50 & 52 & 100 \\
5 & 2 & $1.0 \times 105$ & 40 & 62 & 85 \\
\hline
\end{tabular}

behavior found in homologous series of low molar mass liquid crystalline materials (Lenz, 1985). In these cases, the increase is attributed, at least in a first approach, to the increasing degree of anisotropy of the mesogenic unit. Of course, this is also true of side-chain liquid crystal polymers but for such materials there is the added complications arising from the constraints and interactions provided by the polymer chain. It would be reasonable to assume that as the coupling chain shortened, so, the coupling between the mesogenic unit and the polymer chain would increase. If ordering increases as a result of this coupling, it is natural to expect the transitions to rise. In practice as shown in Table 1. The opposite is observed. This suggests that the emphasis on the role of the polymer chain should be one of constraint than enhanced ordering through coupling. Clearly there is a subtle balance between these possibilities and small variation can lead to marked effects. There is a strong temperature dependence of the switching times. In terms of the variation of the nematicisotropic, variation transition temperature with spacer length, this appears to have some element of the wellknown odd-even effect observed in low molar mass liquid crystalline materials and in main chain liquid crystal polymers (AL-Ammar, 2008). The increase in the glass transition temperature with decreasing spacer length follows expectations based on the restricted motions of the polymer backbone segments. Clear trends are observed in the threshold voltage variation as a function of both temperature and spacer length. The static electric optic properties are important because they contain important experiments between the threshold voltage Uc and curvature elastic constants $\mathrm{K}_{\mathrm{ii}}$ and their relationship to composition and temperature for the liquid crystal copolymers.

In order to assess the curvature elastic constants K11 must be measuring the threshold voltage required to induce director deformation in an electro-optic cell in which the director has a predefined surface alignment. The method of measuring Uc is to determine the smallest voltage required to produce a significant optical change (Blinov, 1983). It is emphasized that considerable care and effort has been taken to exclude from these experiments the time dependent effects of the high viscosities associated with polymeric liquid crystal systems. In part 
the increase of the threshold voltage with decreasing temperature is expected on the basis of an increasing order parameter. The origin of the variation of the threshold voltage with spacer length is not so clear. It seems reasonable to attribute the increase of the intrinsic elastic constant to reducmg the spacer length to changing levels of interactions between the mesogenic units and the polymer backbone.

\section{CONCLUSION}

We have previously reported some threshold voltage measurements for a series of methylmcthacrylatce based side-chain liquid crystal polymers with differing molecular weights.

\section{REFERENCES}

Blinov, L.M., 1983. Electro-Optical and Magneto-Optical Properties of Liquid Crystals. Wiely, Hoboken, New Jersey, USA., ISBN:9780471102311, Pages: 341 .

Coles, H.J., 1988. Electro-Optic Applications of Liquid Crystalline Polymers. In: Developments in Crystalline Polymers, Bassett, D.C. (Ed.). Springer, Dordrecht, Netherlands, ISBN:978-94-010-7096-6, pp: 297-340.

Gennes, D.P.G., 1971. Short range order effects in the isotropic phase of nematics and cholesterics. Mol. Cryst. Liq. Cryst., 12: 193-214.

Haase, W., 1989. Field-Induced Effects. In: Side Chain Liquid Crystal Polymers, McArdle, C.B. (Ed.). Blackie \& Son Glasgow, Scotland, London, UK., ISBN:9780412017612, pp: 309-329.

Leslie, F.M., 1992. Continuum theory for nematic liquid crystals. Continuum Mech. Thermodyn., 4: 167-175.

McArdle, C.B., 1989. Side Chain Liquid Crystal Polymers. Chapman and Hall, Scotland, ISBN:9780412017612, Pages: 451.
McArdle, C.B., 1990. Side Chain Liquid Crystal Polymers. Springer, Berlin, Germany, Pages: 451.

Noirez, L., J.P. Cotton, F. Hardouin, P. Keller and F. Moussa et al., 1988. Backbone conformation of a mesogenic side-chain polyacrylate. Macromol., 21: 2889-2891.

Plate, N.A. and V.P. Shibave, 1987. Comb-Shaped Polymers and Liquid Crystals. Plenum Press Publisher, New York, USA., Pages: 415.

Portugall, M., H. Ringsdorf and R. Zente1, 1982. Synthesis and phase behaviour of liquid crystalline polyacrylates. Macromolecular Chem. Phys., 183: 2311-2321.

Ringsdorf, H. and R. Zentel, 1982. Liquid crystalline side chain polymers and their behaviour in the electric field. Macromol. Chem. Phys., 183: 1245-1256.

Tal'Roze, R.V., V.P. Shibayev and N.A. Plate, 1983. Thermotropic liquid crystalline polymers in electric and magnetic fields. Rev. Polym. Sci. U.S.S.R., 25 : 2863-2888.

Vertogen, G. and W.H. Jeu, 1988. Thermotropic Liquid Crystals, Fundamentals. Sringer, Berlin, Germany, ISBN:9780387179469, Pages: 324.

Vogel, A.I., 1978. Textbook of Practical Organic Chemistry, Including Qualitative Organic Analysis. 4th Edn., Longman, London, UK., ISBN:9780582407411, Pages: 1371.

Wangsoub, S. and G.R. Mitchell, 2009. Shear controlled crystal size definition in a low molar mass compound using a polymeric solvent. Soft Matter, 5: 525-528.

Zentel, R., 1998. Handbook of Liquid Crystals. In: High Molecular Weight Liquid Crystals, Demus, D., J. Goodby, G.W. Gray, H.W. Spiess and V. Vill, (Eds.). Wiley-VCH, Weinheim, Germany, pp: 303-305. 